Article

\title{
Design and Programming of a Micro-controller-Based Solar Tracking System
}

\author{
Saman Jaafar $1,+, \neq(1)$ and Farhad Mahmood ${ }^{2, *}$ \\ 1 Physics department, College of science, University of Halabja ; saman.jaafar@uoh.edu.iq \\ 2 Physics department, College of science, University of Halabja; farhad.mahmood@uoh.edu.iq \\ * Correspondence: saman.jaafar@uoh.edu.iq
}

\begin{abstract}
This paper is regarding design and program an Micro-controller Arduino Uno board by using Arduino software to work as a photo-sensor(Active) single axial solar tracker system(SASTS). A solar panel, two photo-resistors (LDR) in two sides (north/south) of the photo-voltaic(PV) and a servo motor are connected to the Uno board, which is running a code that prepared by Arduino software IDE in advanced then it works as a tracking system. Here, the LDRs send the signal of presence or absence of the light to the board and based on that sent signal the Uno reflects a new signal to the servo motor to rotate and finds the light source. Lastly, the photo-sensor single axis tracker is made while Continuously, the system tries to face the panel to the sun and whilst changing the irradiance intensity it starts searching to find the angle of highest irradiance. Based on results that are extracted from the data, the tracker system significantly boosts the output efficiency of the solar panel. By using the Micro-controller Uno board, LDRs, servo motor and special designed mechanical base, the tracking system is constructed, based on acquired data the influence of the STS on the increasing the solar panel efficiency is more obvious. Significantly, the tracker system rises the efficiency of the PV .
\end{abstract}

Keywords: Solar cell energy; Single Axial Solar Tracking System; Solar cell efficiency; Arduino Uno Board

\section{Introduction}

The high consumption of energy and the industrial revolution lead to some concerns about the environment and energy sources. Particularly, raising the temperature of the earth and limitation with the fossil sources. As a result, the researchers so far tried to highly utilize renewable energies to minimize those issues. The solar cell energy is one of the renewable energy that highly recommended as a green energy source for that situation. As Edmond Becquerel discovered the principle of PV system in 1839 , and due to the fact that PV is not introduce any pollution to the environment, therefore it can be called as a renewable energy [7]. Nowadays, the solar panel is mainly used as a major source to produce electrical energy in most of the countries throughout the world, due to the above mentioned facts. Because, it doesn't have a bad impact on the environment such as air pollution (carbon dioxide) and global warming, also, the source of support is the sun while it is an infinite source of energy [1,2]. While the produced energy is directly change with the intensity of the radiation. The intensity of the sun radiation changes due to deflection angle of irradiance. Thus, The output efficiency of the PV depends on the irradiance angle and irradiance intensity. The best and most effective angle is a normal angle, that means the sun light are normally radiate on the PV. Due to continuously change of the position of sun, the radiation energy will not be high during the day time. Since, the Solar Tracker System (STS) is needed to solve that problem[1]. The STS is expensive because it requires a mechanical base and installation. also, it requires electrical energy to work. Mainly, STS has some types:[1]: 
where $I_{D}$ is direct irradiance, $\theta$ is irradiance angle, and $I_{D N}$ is normal direct irradiance.

- Tracking based on optical sensors and microprocessor (Active)

- Tracking based on date and time (Active)

- Fixed or manually change tracker

Mainly, the Active STS can be divided into Single axial and dual axial tracker. When the single axial STS only tracks solar in azimuth path but can't track solar in altitude path[3]. Strongly, the cost of the tracker's structure (Mechanical base, installation) depends on the amount and type of material that want to be used. Also, the geometry of the structure is important in order to be expensive [4]. Here, the paper seeks to investigate the effect of the STS on the output efficiency of the solar panel.Based on the measured data the efficiency has raised by $20 \%$.

\section{Methodology}

\subsection{Solar Radiation}

Solar radiation is the main key to achieve output efficiency from the PV. The irradiance has two-part, the first is the direct beam, that reached to the PV surface and the second is reflected irradiance which is reflected from the PV surface, in addition to that; it does not provide energy to $\mathrm{PV}$ in order to produce electricity[5]. The direct irradiance received on the PV surface is given by the equation

$$
I_{D}=I_{D N} \cos \theta
$$

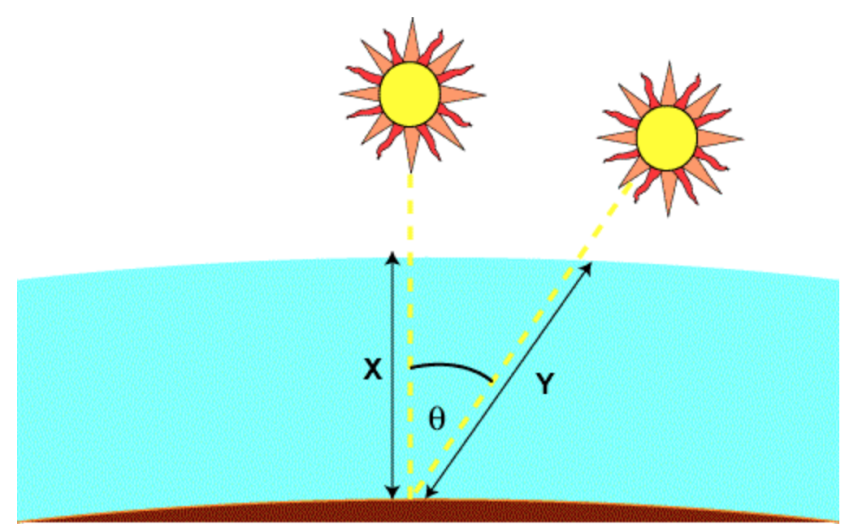

Figure 1. Sun Radiation Angle

Sun position can be determined by two angles: Altitude angle and Azimuth angle. this angle is given by

$$
\alpha=\sin ^{-1}(\cos \phi \times \cos \delta \times \cos \omega+\sin \phi \times \sin \delta)
$$

While the second angle is given by

$$
\cos \gamma=\frac{\sin \alpha \times \sin \phi-\sin \delta}{\cos \alpha \times \sin \phi}
$$

47 Both angles of altitude and azimuth can be calculated by using the information on local attitude, $(\phi)$

48 Solar declination angle, $(\delta)$ and hour angle, $(\omega)[6]$.

\subsection{Design and Equipment}

The mechanical structure of the tracker system is made of Aluminum and it has lightweight with servo motor together about $200 \mathrm{~g}$. the overall volume which may be taken by the structure is about $30 \mathrm{~cm} \times 25 \mathrm{~cm} \times 25 \mathrm{~cm}$. it consists of A structure holder, gear shaft, panel holder and servo motor holder. 
53

54

It could be move in altitude path (West to East) and easily can work as a Single Axial Solar Tracking System(SASTS). the motion of the gear shaft is controlled by the servo motor and it rotates with low torque which can be handle by the servo motor (MG-996R). the main point is that the Uno board can supply the servo motor for rotation with specifying speed and angle. The rigidness and low air resistance are the good points of the mechanical structure of the SASTS.

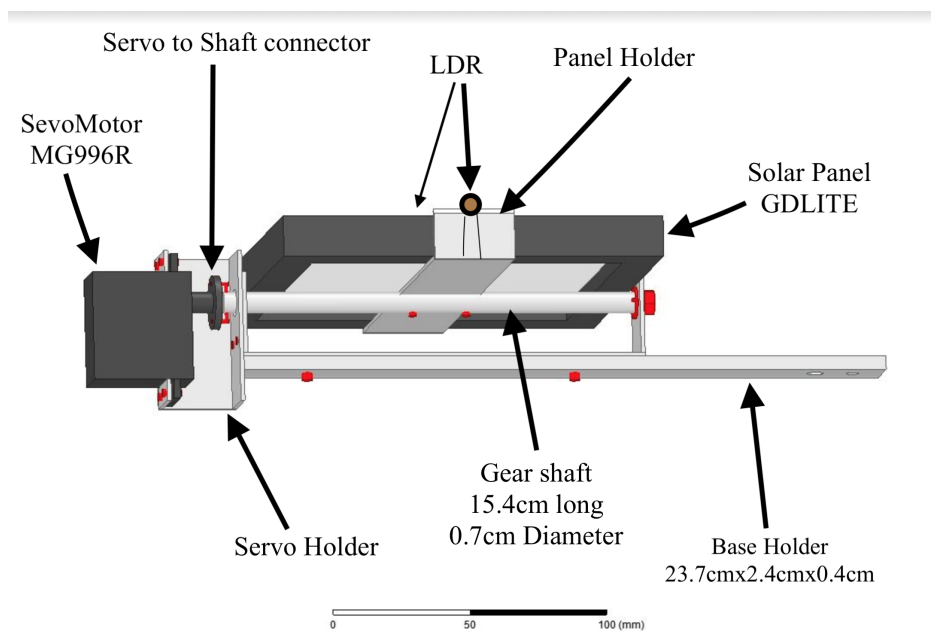

Figure 2. Mechanical Structure of SASTS

\subsection{Uno Board}

The Arduino Uno board is an open-source micro-controller[12]. It has sets of digital and analog Input/Output(I/O) pins, here,as shown in the Figure 3, the digital pins of 2,3,4,5 which working as output pins are sending the signals to switching on/off the MOSFETs one by one and in pair and triple and all together. also, the analog pins such as A0,A1 get the signal from the LDR's and A3, A4, A5, and A6 get the output voltages from the switching circuit and these pins work as input pins. the micro-controller also has USB type B which can connect to the computer and can load with Arduino IDE (Integrated Development Environment). It can be powered by a voltage between 9-20V and all digital and analog pins support 5V voltage [13].

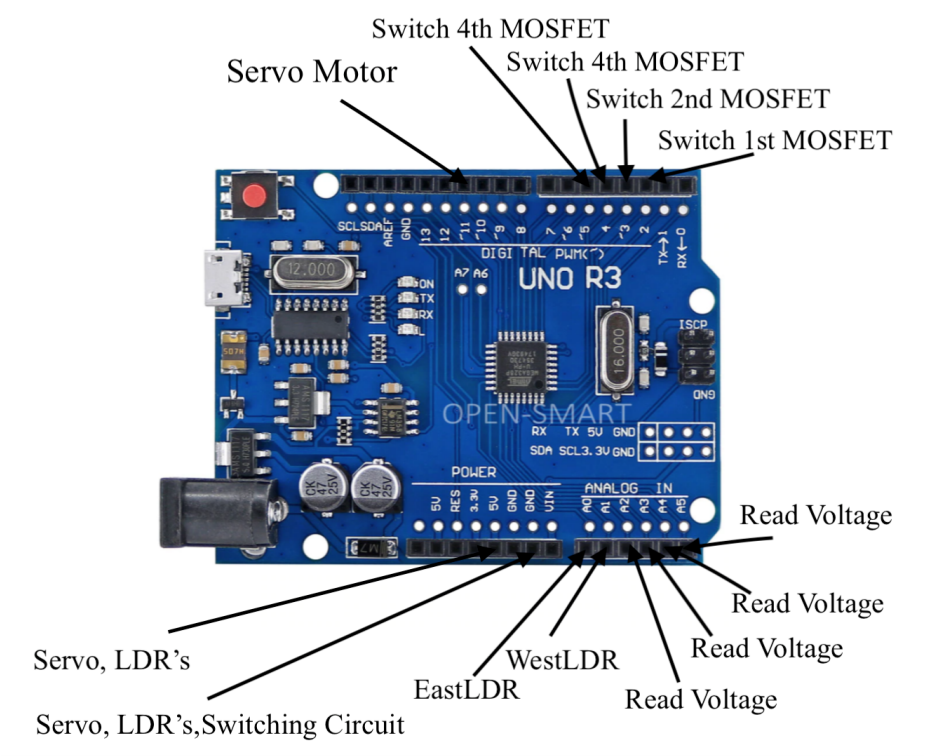

Figure 3. Micro-controller Uno Board 
LDR is abbreviation from Light Dependent Resistance, it made from semiconductor materials and its resistance depend on the intensity of the light, by increasing the light the resistance will decrease and wise versa[5]. Here, the LDR working as photo-sensor and two LDR's are used and when the light intensity be different on them, their resistances can not be equal, like Figure 4 and the circuit would be unbalanced, then, they will send a signal to Uno board and based on the written code in the micro-controller a signal send to the servo motor to rotate until the LDR's circuit to be balanced again[14].

$$
\begin{aligned}
R_{L D R 1} & =\frac{R_{1}}{\frac{V_{s}}{V_{L D R 1}}-1} \\
R_{L D R 2} & =\frac{R_{2}}{\frac{V_{S}}{V_{L D R 2}}-1}
\end{aligned}
$$

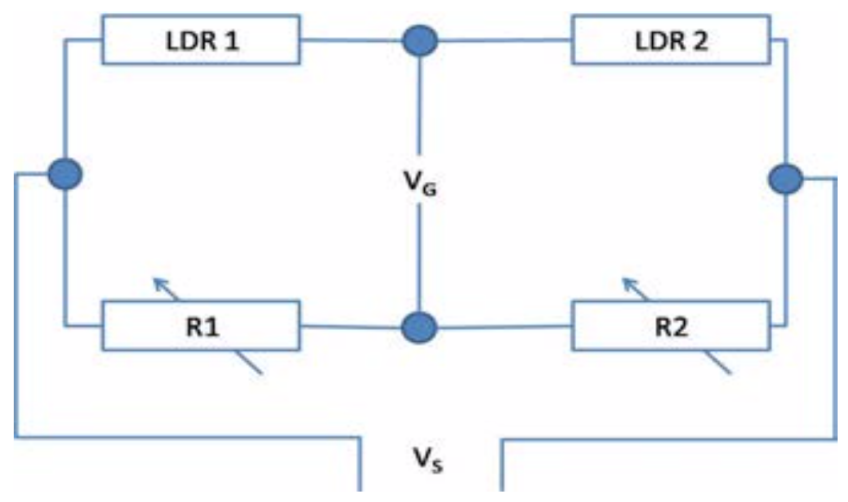

Figure 4. Balance Circuit

From Equations 4 and 5, if $R_{1}=R_{2}$ and $V_{L D R 1}=V_{L D R 2}=V_{g}$ and then $R_{L D R 1}=R_{L D R 2}$ while balance mode, as mentioned before, the resistance value of each LDR depends on the intensity of the light, therefore, this will produce unbalance system if the intensity of the light change too. This phenomenon helps to make a photo-sensor system that continuously searches for maximum intensity to have a balance between both east-LDR and west-LDR.

\subsection{Servo Motor}

This High-Torque Metal Gear Servo Motor for Robotics(MG996R) Digital Servo can rotate approximately 120 degrees (60 in each direction) and can support about $10 \mathrm{Kg}$ load. Meanwhile, any servo code, hardware, or library to control these servos can be used[15]. A servomotors are rotary or linear actuator those allow for precise control of angular or linear position, velocity and acceleration [8]. Therefor based on the output signal which comes from the Uno board to servo motor and then the motor starts to rotate, bearing in mind the weight of the solar panel is about $200 \mathrm{~g}$ and simply the servo can withstand that amount of load. The main benefits of the servo motor is to supply voltage which is from $4.8 \mathrm{~V}$ to $7.2 \mathrm{~V}$ and this amount can be support by the Uno board. 

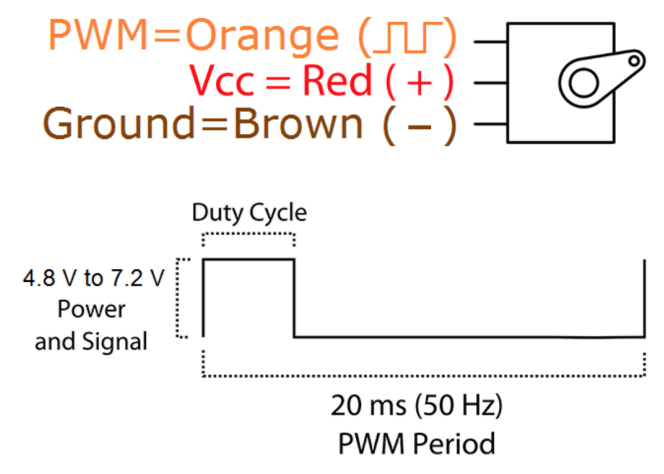

Figure 5. PWM Period,Duty cycle and Power \\ 2.6. Switching Interface Circuit (Voltage regulator circuit)}

The switching interface circuit used for some purposes, the input voltage from the solar panel is around $12 \mathrm{~V}$ and this amount of voltage is high for microcomputer to read, as it has been mentioned before those pins can support maximum $5 \mathrm{~V}$ in both digital and analog. Accordingly, the voltage divider board is designed by $R_{5}$ to $R_{10}$ and the regulated voltage would be directed toward Uno board through the outgoing signal from the digital pins. The aim of using of $R_{1}, R_{2}, R_{3}, R_{4}$ are just for protection of MOSFET's. The MOSFET's work based on the digital pins of the Uno board, while the micro-controller would respond based on the signal from the written code on the board. Additional purpose of designed circuit is to have several different voltages. Each of the MOSFETs can be turned ON by the digital pins of the micro-controller and each time Uno receive one voltage data, once,the first switching mode has been finished then the new as a switching modes will emerge such as double transistors, triple and quadruple based on the aforementioned modes the Uno board can receive about 14 different voltage measurements. Based on Kirchhoff's Voltage Law (KVL), the input voltage (V-pv) and the voltage on $R_{5}$ and $R_{10}$ is

$$
V_{p v}=V_{R_{5}}+V_{R_{10}}
$$

Both resistors $\left(R_{5}\right.$ and $\left.R_{10}\right)$ are connected in series, thus, based on Kirchhoff's Current Low (KCL) the amount of current which passes through each resistors are same so, equation 6 can be rearrange as shown in equation 7 :

$$
V_{p v}=I R_{5}+I R_{10}
$$

The output voltage which is a part of the total resistance and can be determined as equation 8 .

$$
V_{o}=\frac{R_{10}}{R_{5}+R_{10}} x V_{p v}
$$

The output voltage of solar panel is $11.25 \mathrm{~V}$ and $R_{5}=147 \mathrm{~K} \Omega$ and $R_{10}=100 \mathrm{~K} \Omega$, while, the output voltage of the Uno board can be determined by substituting the values in to equation 8 as depicted in equation 9.

$$
V_{o}=\frac{100}{100+147} \times 11=4.45 \mathrm{~V}
$$

The acquired output voltage (4.45V)is a satisfactory value for the input to the micro-controller. That process of determination of output voltage of switching interface board will be repeated for all other resistors. 


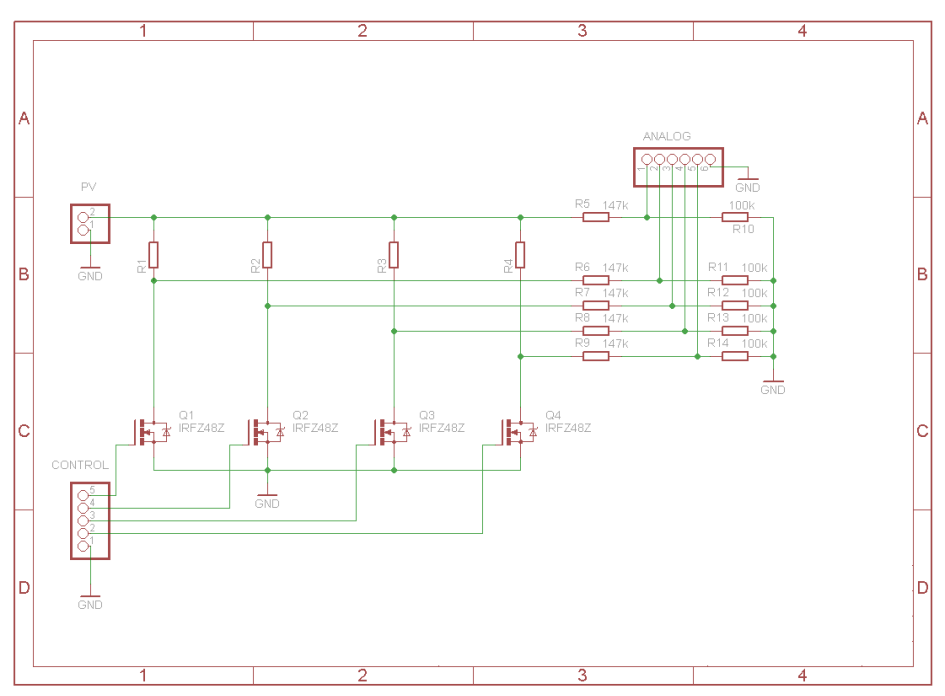

Figure 6. Switching Interface Circuit

$86 \quad$ 2.7. Solar Panel

${ }_{87} \quad$ The mechanical base and servo motor can easily manage the amount of the solar panel's weight 88 due to the specification of the panel since it has light weight and the panel has the following s9 specifications:

Table 1. Specification of GD-020WP Solar Panel

\begin{tabular}{|c|c|c|c|}
\hline Parameters & Current/A & Volatge/V & Power/W \\
\hline Peak Power & & & 2 \\
\hline Open Cicuit Voltage & 0.267 & & \\
\hline Short Circuit Current & & 11.25 & \\
\hline Maximum Power Voltage & & 9 & \\
\hline Maximum Power Current & 0.23 & & \\
\hline
\end{tabular}

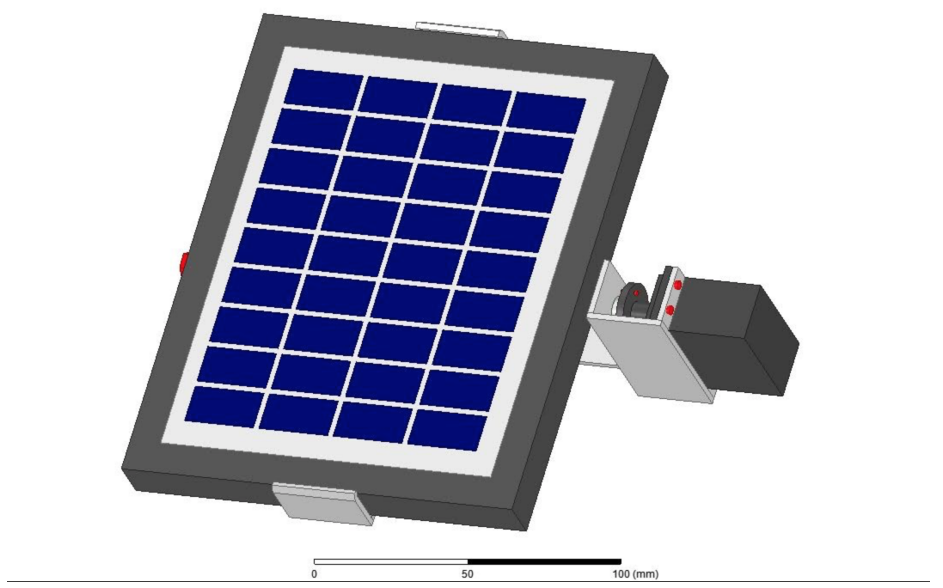

Figure 7. Solar Panel GD-020WP

\section{Integrated System}

The SASTS came from the combination of the micro-controller Uno board, LDR's, servo motor, switching interface circuit, and a solar panel, the system would be weighted around $1 \mathrm{Kg}$, and can be installed on the roof, wall, ground and it could be fastened by two screws. The switching circuit board, 
94 958 .

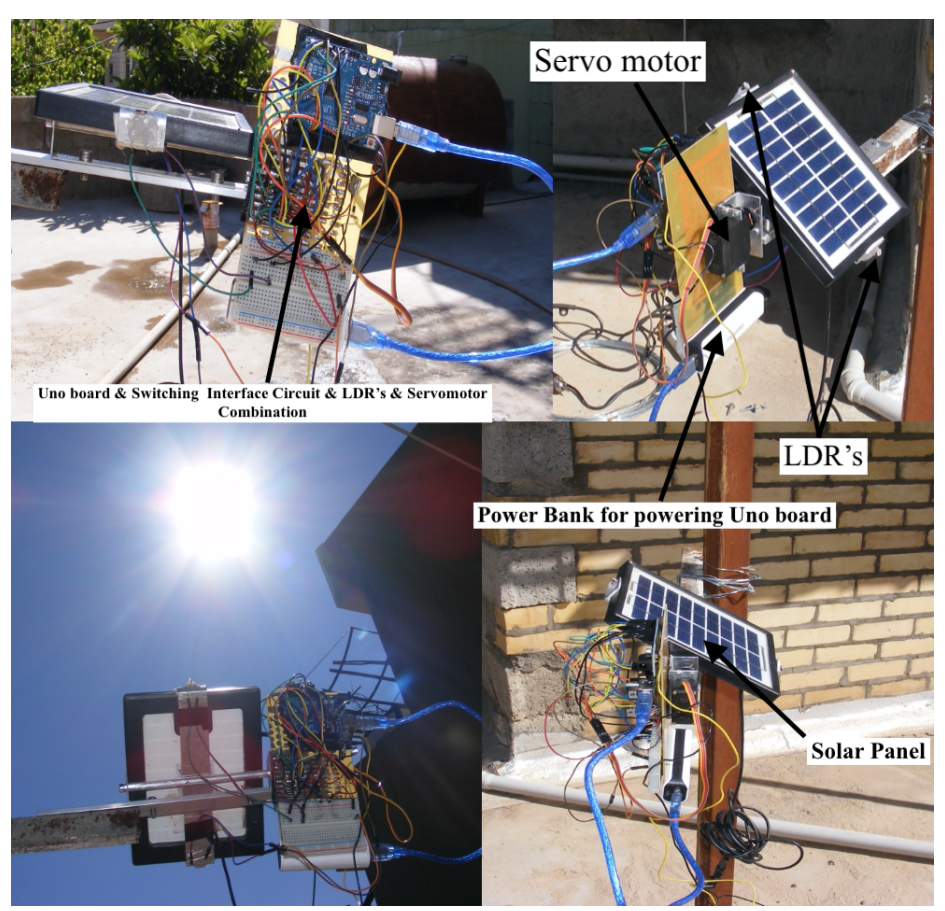

Figure 8. The Tracker System in Different Angle of View

the Uno board and portable battery are attached to the body of the mechanical base as shown in Figure

\section{Flow chart}

The process of the written code in the Arduino Uno board can represented as below flow chart. As it is shown, the tracker system mainly depend on the LDR's signals and the amount of error can be calculated by subtracting the value of westLDR from westLDR. while the intensity of light in one side of solar panel is greater than the other side, then an amount of error will be produced and it would be sensed by Uno board, therefore the Uno board sends a signal to the servo to rotate in the direction of higher intensity LDR and the panel should be adjusted accordingly,then the Uno board recheck the LDR intensities if still the Uno board sensed an amount of the error which is be greater than absolute value of 5 Lux then the panel will rotate accordingly, but, if the error is smaller than absolute value of 5 Lux the system would received the voltages data as explained in the section of Switching Interface 
Circuit.

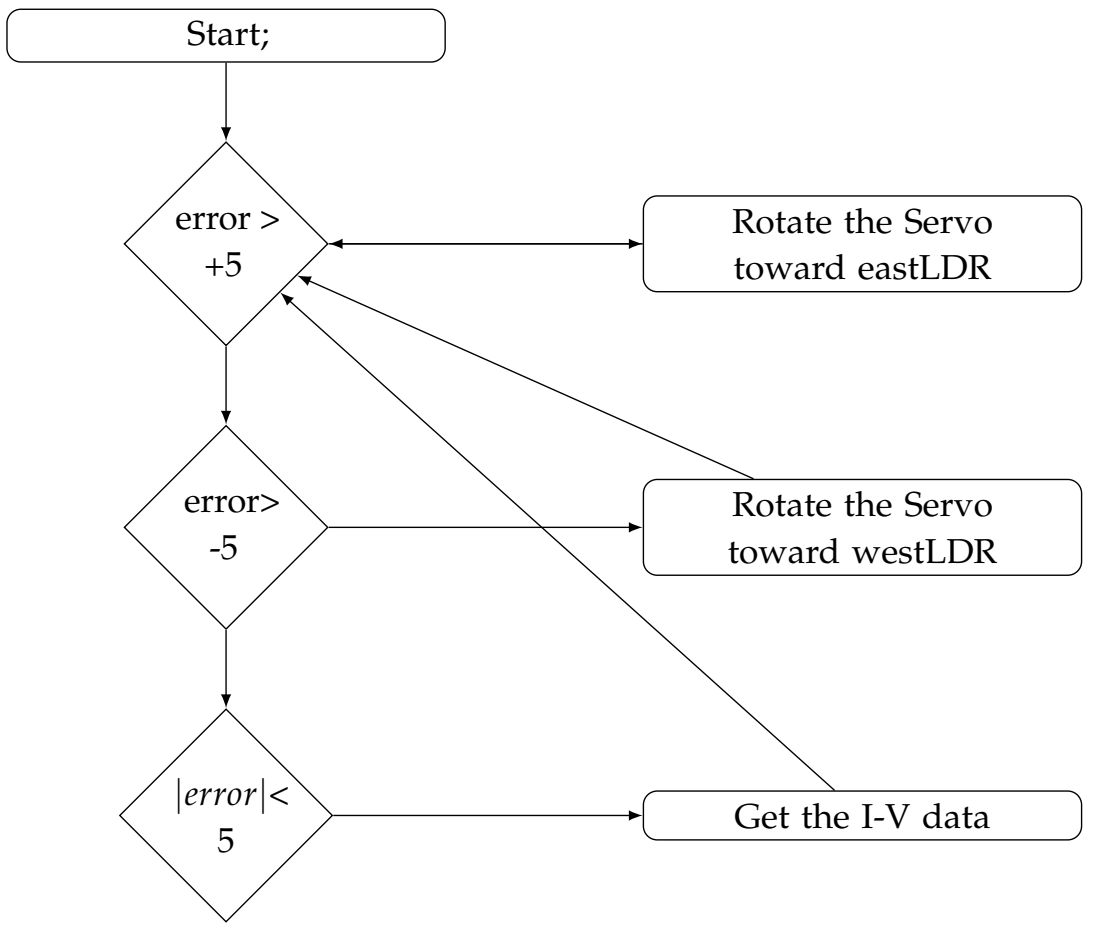

\section{Operation of SASTS}

LDR's are the eyes of the system. They try to find the sun in the sky and prepare the solar panel to be fixed while incoming normal radiation as proved in equation 4,5 , because the most effective irradiance is that rays which normally incident on the PV based on equation 1 . The LDR's lie in both sides of the PV as shown in Figure 2. the incoming a notification or a signal from LDR's to the Uno board makes a change in the decision of the board and it will decide based on the new happened event and send a signal to the servo motor to rotate in the angle toward the highlighted LDR, while doing this process and rotating the servo as a result the new signal will come to the Uno board again from the LDR's and the Uno board specify the value of error, if the error value is smaller than the value which has been assumed, the Uno sends a new signal to the servo motor again and stop the rotation. If the error is larger than the specified value the new signal is going to continue the rotation. The Uno board will get the voltage measures from the switching interface circuit while the error is small. Therefore, only voltage data will be measured, but, based on the code the uploaded to the Uno bard, the current and power for each data will be derived by the known resistors which have been used in the circuit.

\section{Experimental detail}

After finishing the Single Axial Solar Tracker System (SASTS) it can be used for determine the Maximum Power Point Tracking based on the data measurements. The system is used at the end of April 2020 for two days from 8:00am to 16:00 in Halabja city in Kurdistan region/Iraq. The first day the SASTS is working and the data have been collected while using photo-sensor tracker. The data are taken for each one hour its why there are some samples of the data that the prevalent has taken. In the second day the system used but without tracker system, it is like fixed solar panel. The aim of this two different situation is for proof and determine the influence of the tracker on boosting of the efficiency of the solar panel. 
131

Table 2. I-V-P Data in Fixed Solar Panel

\begin{tabular}{|c|c|c|}
\hline Voltage/V & Current/mA & Power/mW \\
\hline 0 & 4.1 & 0 \\
\hline 2 & 4 & 8 \\
\hline 4 & 3.9 & 15.6 \\
\hline 6 & 3.8 & 22.8 \\
\hline 8 & 3.6 & 28.8 \\
\hline 9 & 3.5 & 31.5 \\
\hline 9.5 & 3.1 & 29.45 \\
\hline 9.8 & 2.5 & 24.5 \\
\hline 10 & 1.8 & 18 \\
\hline 10.1 & 1 & 10.1 \\
\hline 10.2 & 0.3 & 3.03 \\
\hline
\end{tabular}

\section{Experimental results}

As discussed before, the Uno board is the heart of the system. Based on the written code by Arduino IDE it takes responsibility for any coming situation. The data have been achieved from the experiments and shown in both Tables 2, 3 and Figures 9a 9b ,10a 10b. Here, for the case of the fixed solar panel, the data have been collected in the different deflection angles of sun radiation ( 0 to $90 \mathrm{deg}$ ) and the average value is chosen (neither the best normal irradiance and nor the worst, 90deg deflection). The Maximum Power Point has been derived from the voltage-Power curve as 10 for both cases of the experiments.

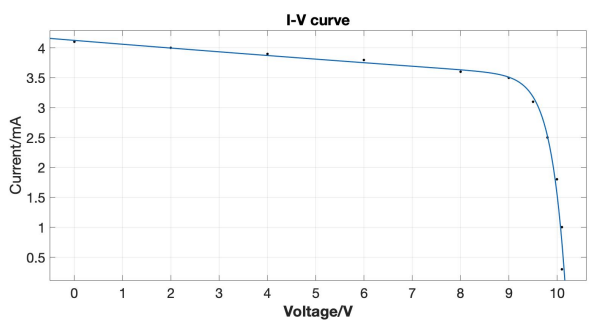

(a) I-V Curve

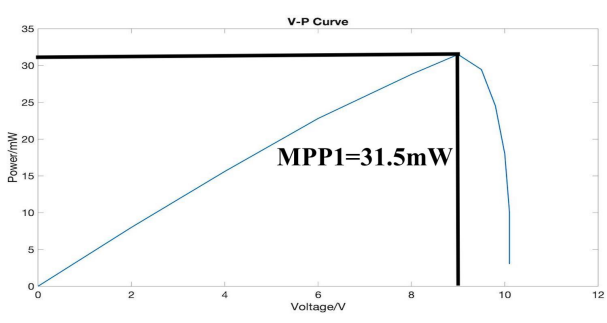

(b) V-P Curve

Figure 9. The Solar Panel Characterization in Fixed Solar Panel

Table 3. I-V-P Data by Using SASTS

\begin{tabular}{|c|c|c|}
\hline Voltage/V & Current/mA & Power $/ \mathrm{mW}$ \\
\hline 0 & 90 & 0 \\
\hline 2 & 87 & 174 \\
\hline 4 & 85 & 340 \\
\hline 6 & 80 & 480 \\
\hline 8 & 78 & 624 \\
\hline 9 & 75 & 675 \\
\hline 9.5 & 67 & 636 \\
\hline 9.8 & 60 & 588 \\
\hline 10 & 40 & 400 \\
\hline 10.1 & 30 & 303 \\
\hline 10.2 & 10 & 102 \\
\hline
\end{tabular}




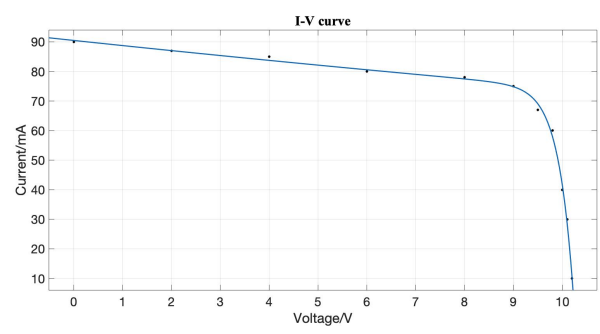

(a) I-V Curve

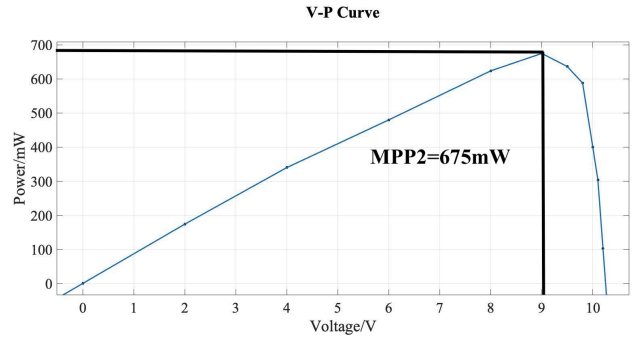

(b) V-P Curve

Figure 10. The Solar Panel Characterization by Using SASTS

\section{Efficiency}

In this section the effective of the SASTS can find and understand on the improving the efficiency of the PV. So, the boost of efficiency is determined by the ratio of the achieved MPP point by using SASTS to the MPP point is obtained from a fixed solar panel. The MPP2 is obtained from using STS and MPP1 from the fixed case, the efficiency can be determining as below:

$$
\eta=\frac{M P P 2}{M P P 1} \times 100 \%
$$

where $\eta$ is efficiency $M P P 2=675 \mathrm{~mW}$ with STS achieved from the graphs, for a fixed case as shown in the $9 \mathrm{~b}$, there are fourteen MPP points but any of them are related to the specific deflection angle. Therefore, the Maximum and the Minimum of the MPP can be focused, that means the biggest and smallest angle of deflection. $M P P 1=31.5 \mathrm{~mW}$ Now, just we will substitute the values in equation 10 . $\eta=\frac{M P P 2}{M P P 1} \times 100 \%=\frac{675}{31.5} \times 100 \%=21.42 \%$

\section{Comparison}

The comparison section will make fine clarification for all the performed tasks. From the plotted data in figure $9 \mathrm{~b}$ for the fixed case of solar panel, the MPP is low due to a high deflection angle. Based on equation 1 the highest intensity is obtained form the 0 angles of deflection of the sun's rays. Its the main reason for low achieved MPP in that case. Meanwhile, by using SASTS, the plotted data in the Figure $10 \mathrm{~b}$ were shown that the high amount of the solar radiation has been converted to the electrical energy, because, the MPP point is higher than the previous case. Definitely, it can be said that the main reason for that is the minimization of the deflection angle of radiation by the solar tracker system. The MPP is about 21 times greater than the MPP of the fixed case, thus the solar tracker (photo-sensor tracker) is so effective on boosting the output energy for the PV system.

\section{Conclusion}

Significantly, using SASTS caused to achieve more power from the solar panel and provide to boost efficiency. It is a good idea to use the tracker for larger projects because based on the determined efficiency due to using the SASTS about $21.4 \%$ rising of the efficiency has achieved. The advantages of the solar tracker can be mention as below points.

$\star$ The SASTS is a system that needs low energy to work and is supported by the Uno board. But, for two-axial STS would need more electrical energy to handle.

$\star$ Here, the system can be handled by the output energy of the solar panel. Therefor, the working of the STS can be done by the self-support of the solar panel

$\star$ Advancements in technology and reliability in electronics and mechanics are gradually declining the long-term maintenance concerns about STS [11].

$\star$ It is highly recommend for those geographical locations which don't have more time of Sun 
irradiance or the intensity of the radiation is poor, means the sun radiate by the high deflected angle (usually north part of the earth and far locations from equator line).

* STS caused to speed up the charging of the battery, the battery charging almost depends on the power and efficiency of the PV. So, when the efficiency of the PV increased the charging of the battery will be faster. Thus, the battery will not going to die sooner than its lifetime.

There are some drawbacks of the SASTS as discussed below:

$\star$ The SASTS may not be able to track all sun motion paths thus, some of the radiation time will be wasted and it will not be used and the output efficiency will be less than the peak value.

$\star$ It needs energy to work, for a large project, a large amount of energy needs to handle the process

$\star$ For its utilization, the wide and flat ground is needed. That Means, a suitable place is required to put the system on it. Meanwhile, the place must be wide and sufficiently far from the trees and the agent of shadow .

$\star$ The construction of the mechanical base of the tracker needs cost, tools, and time. The tracker also needs observation and maintenance.

\title{
11. Future work
}

Some points about the future of this project:

$\star$ Do the project and the experiments in the northern area of the earth because there the deflection angle of the incident ray is more and the influence of the using solar tracker system can be more understandable.

$\star$ Use higher power solar panel since, MPP points are more sensible during finding.

$\star$ Do the same procedure with two axial solar tracker system, it maybe the effective of the tracker will be highly observe.

$\star$ Using MATLAB Simulink for doing this kind of procedures.

\author{
Abbreviations \\ The following abbreviations are used in this manuscript: \\ SASTS Single Axial Solar Tracker System \\ MPP Maximum Power Point \\ PV Photo-Voltaic \\ LDR Light Depending Resistor \\ IDE Integrated Development Environment
}

\section{References}

1. F. M. Hoffmann, R. F. Molz, J. V. Kothe, E. O. B. Nara, and L. P. C. Tedesco, “Monthly profile analysis based on a two-axis solar tracker proposal for photovoltaic panels," Renew. Energy, vol. 115, pp. 750-759, 2018.

2. T. Hong et al., "A preliminary study on the 2-axis hybrid solar tracking method for the smart photovoltaic blind," Energy Procedia, vol. 88, pp. 484-490, 2016.

3. J. Song, Y. Zhu, D. Xia, and Y. Yang, "A photovoltaic solar tracking system with bidirectional sliding axle for building integration," Energy Procedia, vol. 61, pp. 1638-1641, 2014.

4. H. Zlatanov and G. Weinrebe, "CSP and PV solar tracker optimization tool," Energy Procedia, vol. 49, pp. 1603-1611, 2013.

5. D. F. Da Silva and D. Acosta-Avalos, "Light dependent resistance as a sensor in spectroscopy setups using pulsed light and compared with electret microphones," Sensors, vol. 6, no. 5, pp. 514-525, 2006.

6. S. Ahmad, S. Shafie, and M. Z. A. Ab Kadir, "Power Feasibility of a Low Power Consumption Solar Tracker," Procedia Environ. Sci., vol. 17, pp. 494-502, 2013.

7. A. Goetzberger and A. Goetzberger, "Workshop on " Physics for Renewable Energy " Crystalline Silicon Solar Cells Crystalline Silicon Solar Cells," 2005. 
212 8. L. Szabó and A. Szaniszló, "Monthly profile analysis based on a two-axis solar tracker proposal for 213 photovoltaic panels," Int. Rev. Appl. Sci. Eng., vol. 8, no. 1, pp. 37-43, 2017.

214 9. S. Sugiyama, "Multi-Stage PWM DC Servo Motor Control," IFAC Proc. Vol., vol. 22, no. 18, pp. 341-345, $215 \quad 2017$.

216 10. L. Louis, “SOLAR POWER PLANT STATION IN FISHLAND,” Int. J. Control. Autom. Commun. Syst., vol. $217 \quad 1$, no. 2, pp. 21-29, 2016.

218 11. "Solar power world.pdf." , Advantages and disadvantages of Solar Tracker system, 219 https://www.solarpowerworldonline.com/2016/05/advantages-disadvantages-solar-tracker-system/

220 12. "What is Arduino?". learn.sparkfun.com. Retrieved 4 February 2018

221 13. "Arduino". store.arduino.cc. Retrieved 10 March 2020.

14. "Photoresistor" https : //www.electronics - notes.com/articles/electronic components/resistors/light 223 dependent - resistor - ldr.php. "MG996R High Torque Metal Gear Dual Ball
//www.electronicoscaldas.com/datasheet/MG996R T $_{\text {ower }}-$ Pro.pdf 